Revista Med 26(2) 2018

\title{
ASOCIACIÓN DIABETES TIPO 2 Y TUBERCULOSIS PULMONAR EN PACIENTES DE IMSALUD EN LA ZONA ATALAYA DE CÚCUTA ENTRE 2013 Y 2015
}

\author{
RUBY LORENA LOZADA MUÑOZ1, OSSKAR IVÁN RINCÓN ARDILA², \\ YALEISY ROMÁN RAMÍREZ3 \\ ${ }^{1}$ Universidad de Pamplona, Colombia. \\ 2 Universidad de Pamplona, Colombia. \\ ${ }^{3}$ Universidad de Pamplona, Colombia. \\ Correspondencia: Osskar Iván Rincón Ardila \\ ivan25r@hotmail.com
}

Recibido: 29 de marzo de 2018 Aceptado: 5 de junio de 2018

\section{Resumen}

Objetivo: Establecer la asociación entre diabetes mellitus tipo 2 (DM2) y tuberculosis pulmonar (TBp) en los pacientes que asisten al servicio de consulta externa en las IPS de Imsalud de la Zona Atalaya de Cúcuta, Norte de Santander. Material y métodos: Se realizó un estudio observacional, analítico, de tipo casos y controles, en que se seleccionaron a todos los pacientes que asistieron a las IPS de Imsalud de la Zona Atalaya de enero de 2013 a diciembre de 2015 (28 015 pacientes). Se revisaron las historias clínicas de los pacientes con diagnóstico de TB (100) y se obtuvo el consolidado de pacientes diabéticos (847) a partir de la base de datos de Imsalud. Resultados: De una población total de 28 015 pacientes, 100 (0,4\%) y 847 (3\%) tenían diagnóstico de TBp y DM2, respectivamente. De los 100 pacientes con tuberculosis, $17(0,1 \%)$ tenían a la vez diagnóstico de DM2. La asociación estadística (chi cuadrado) para las variables DM2 y TBp reportó un resultado de 66,8 para una p: $<0,05$ (IC: 5,1-8,3). Conclusiones: La literatura plantea que existe mayor susceptibilidad en las personas con DM2 de contraer TBp y que esta puede ser explicada por defectos en la inmunidad celular, causados por los periodos de hiperglicemia, además de la alteración de las funciones de los leucocitos polimorfonucleares. En este estudio, se estableció una asociación significativa del binomio DM2-TBp, que reporta que existe un riesgo (OR) 6,7 veces mayor de desarrollar TBp en pacientes con antecedente de DM2 que en aquellos que no presentan esta comorbilidad.

Palabras clave: tuberculosis pulmonar; diabetes mellitus tipo 2; asociación; inmunidad; observacional 


\title{
ASSOCIATION OF TYPE 2 DIABETES MELLITUS AND TUBERCULOSIS IN IMSALUD PATIENTS IN THE ATALAYA ZONE OF CUCUTA 2013-2015
}

\begin{abstract}
Objective: To establish the association between type 2 diabetes mellitus (DM2) and pulmonary tuberculosis (TBp) in patients attending the outpatient service in the Health Care Provider (IPS from "Institución Prestadora de Salud" in Spanish) of Imsalud of the Atalaya Zone in Cucuta, Norte de Santander. Material and methods: An observational, analytical, case-control study was conducted, in which all the patients who attended the IPS of Imsalud in the Atalaya Zone from January 2013 to December 2015 (28,015 patients) were selected. The medical records of patients with a diagnosis of TB (100) were reviewed and the consolidation of diabetic patients (847) was obtained from the Imsalud database. Results: Out of a total population of 28,015 patients, $100(0.4 \%)$ and 847 (3\%) had a diagnosis of TBp and DM2, respectively. Out of the 100 patients with tuberculosis, $17(0.1 \%)$ also had a diagnosis of DM2. The statistical association (chi square) for the variables DM2 and TBp reported a result of 66.8 for a p: $<0.05$ (CI: 5.1-8.3). Conclusions: The literature suggests that there is greater susceptibility in people with DM2 to contract TBp and that this can be explained by defects in cellular immunity, caused by periods of hyperglycemia, in addition to the alteration of the functions of polymorphonuclear leukocytes. In this study, a significant association of the DM2-TBp binomial was established, which states that there is a 6.7 times greater risk (OR) of developing TBp in patients with a history of DM2 than in those who do not have this comorbidity.
\end{abstract}

Keywords: pulmonary tuberculosis; diabetes mellitus type 2; association; immunity; observational

\section{ASSOCIAÇÃO ENTRE DIABETES MELLITUS TIPO 2 E TUBERCULOSE EM PACIENTES DE IMSALUD NA ZONA ATALAYA DE CÚCUTA 2013-2015}

\section{Resumo}

Objetivo: estabelecer a associação entre diabetes mellitus tipo 2 (DM2) e tuberculose pulmonar (TBp) nos pacientes que vão ao serviço de consulta externa nas IPS de Imsalud da Zona Atalaya de Cúcuta, Norte de Santander. Material e métodos: realizou-se um estudo observacional, analítico, de tipo casos e controles, em que se selecionaram todos os pacientes que foram às IPS de Imsalud da Zona Atalaya de janeiro de 2013 a dezembro de 2015 (28 015 pacientes). Revisaram-se as histórias clínicas dos pacientes com diagnóstico de TB (100) e obteve-se o consolidado de pacientes diabéticos (847) a partir do banco de dados de Imsalud. Resultados: de uma população total de 28 015 pacientes, $100(0,4 \%)$ e 847 (3\%) tinham diagnóstico de TBp e DM2, respectivamente. Dos 100 pacientes com tuberculoses, $17(0,1 \%)$ tinham ao mesmo tempo diagnóstico de DM2. A associação estatística (qui-quadrado) para as variáveis DM2 e TBp apresentou um resultado de 66,8 para um p: $<0,05$ (IC: 5,1-8,3). Conclusões: a literatura propõe que exista maior suscetibilidade nas pessoas com DM2 de contrair TBp e que esta pode ser explicada por defeitos na imunidade celular, causados pelos períodos de hiperglicemia, além da alteração das funções dos leucócitos polimorfonucleares. Neste estudo, estabeleceu-se uma associação significativa do binômio DM2-TBp, que mostra que existe um risco (OR) 6,7 vezes maior de desenvolver TBp em pacientes com antecedente de DM2 que nos que não apresentam essa comorbidade.

Palavras-chave: tuberculose pulmonar; diabetes mellitus tipo 2; associação; imunidade; observacional. 


\section{Introducción}

Existe una relación estrecha entre la salud del ser humano, los cambios en el medio ambiente y los avances en la ciencia. Estos últimos han permitido el control y, en algunos casos, la erradicación de ciertas enfermedades. Sin embargo, la existencia de hábitos conductuales y dietéticos no saludables en los individuos impactan la transición epidemiológica de las enfermedades.

Por otra parte, el advenimiento de vacunas, el uso de antibióticos y la implementación de medidas preventivas específicas (1), tales como el uso de protección personal (uso de tapabocas o mascarillas respiratorias) y la filtración del aire para extraer partículas infecciosas en ambientes hospitalarios, constituyen un paso importante en la disminución de la incidencia y mortalidad por enfermedades infecciosas.

Tal es el caso de la TB, una enfermedad infectocontagiosa, oportunista y curable; su principal agente etiológico es el Mycobacterium tuberculosis que se transmite de una persona a otra a través de gotitas generadas en el aparato respiratorio de los pacientes con enfermedad pulmonar activa. Se ha documentado su presencia en diferentes órganos o tejidos (ojos, pleura, riñón, hígado, intestino, peritoneo, entre otros), de modo que es el pulmón su principal localización (2). Afección de alta distribución mundial, una de las enfermedades de mayor importancia desde el punto de vista de la salud pública (3).

El Informe mundial sobre la tuberculosis 2014(13) estimó 9 millones de casos nuevos de TB (de 8,6 millones a 9,4 millones) a nivel mundial, equivalente a 126 casos por cada 100000 hab. La mayor parte del número considerado de casos en 2013 se reportaron en Asia (56\%) y África (29\%); tres proporciones menos de los casos se notificaron en el Mediterráneo oriental (8\%), Europa (4\%) y las Américas (3\%) (4).

En Colombia, en 2013 se notificaron al Sistema de Salud Pública (Sivigila) 12062 casos de TB, de los cuales el $80,8 \%$ pertenecieron a TB pulmonar, el $14,6 \%$ a TB extrapulmonar y el $4,5 \%$ a TB meníngea. Durante este año se reportaron 10849 casos nuevos de TB, para una proporción de incidencia de 23,02 casos por 100000 hab. Para 2015, se reportaron 12918 casos de TB, de los cuales 11647 corresponden a casos nuevos; la incidencia de TB para el país a este periodo es de 24,2 casos por 100000 hab. (5).
A nivel mundial, el número absoluto de casos nuevos de TB muestra una tendencia a la disminución, a una tasa promedio del $1,5 \%$ entre 2000 y 2013 y del $0,6 \%$ entre 2012 y $2013(4)$.

No obstante, se ha observado un incremento estadístico en el número de enfermedades crónicas/degenerativas, como la diabetes mellitus tipo 2 (DM2), que se define como una alteración metabólica, caracterizada por una elevada concentración de glucosa en sangre (hiperglucemia), alteración en la utilización y el aprovechamiento de los carbohidratos, grasas y proteínas provenientes de los alimentos. Además, se presenta resistencia a la acción de la insulina por parte de los tejidos periféricos e inflamación crónica que favorece la disminución de células del sistema inmunitario en presencia de lesiones inflamatorias (2). Por consiguiente, este tipo de enfermedades se establecen como posible factor de riesgo para ciertas enfermedades reemergentes, lo que constituye un gran problema de salud pública.

En la actualidad, la población que padece diabetes mellitus (DM) presenta comportamientos con tendencia al aumento a nivel local, nacional y global (6). El Observatorio Nacional de Salud (7) reportó que entre 2010 y 2014 en Colombia la prevalencia de DM aumentó del 3,4 al 3,9\%. Los cinco departamentos que registraron la prevalencia más alta en ambos sexos y ambos años comparados fueron Valle del Cauca (4,5-5,2\%), Norte de Santander (4,1-4,9\%), Risaralda y Santander (3,9-4,6\%) y Antioquia $(3,8 \%-4,5)$.

En el ambiente hospitalario, es común la implementación de medidas de aislamiento y protección, para pacientes con enfermedades infecciosas, por constituirse en un factor de riesgo para padecer otras enfermedades que agraven el estado de salud e incluso amenacen su vida. Una de las enfermedades que requiere aislamiento es la TBs, la cual cuenta con un protocolo de tratamiento específico e implementación de medidas de protección con el fin de disminuir su evolución e incidencia, respectivamente.

En los pacientes con DM, se implementan medidas terapéuticas iniciales dirigidas a modificar sus hábitos alimenticios, prácticas de ejercicio físico y mantenimiento de un adecuado peso corporal. Sin embargo, en un paciente con mal control de su enfermedad, se genera un estado de depresión del sistema inmunitario, situación que favorece el desarrollo de múltiples infecciones, de modo que es el pie diabético la complicación más frecuente, que a 
su vez puede desencadenar en una amputación, de no ser tratado oportuna y adecuadamente; esto implica especial atención en el cuidado de los pies.

Recientemente, se ha planteado que este grado de susceptibilidad inmunológica también puede incrementar el riesgo de desarrollar infecciones de tipo respiratorio, como la TB. Se han encontrado estudios realizados en América Latina, en países como México (8), Chile (9) y Perú (10), los cuales han concluido que existe una asociación estadísticamente significativa. En Colombia, en una investigación realizada en la ciudad de Bogotá, también se encontró una asociación significativa entre ambas enfermedades (11), lo que sugiere que hay mayor predisposición en pacientes diabéticos de desarrollar TB frente a los no diabéticos.

Teniendo en cuenta que, según la distribución nacional, Norte de Santander se ubica como uno de los departamentos con mayor prevalencia de DM y además alta incidencia de $\mathrm{TB}$, se pretende establecer si existe o no asociación entre estas dos enfermedades, en pacientes que asisten a las IPS de Imsalud en parte de la Zona Atalaya de Cúcuta, entre 2013 y 2015.

\section{Materiales y métodos}

El presente estudio es observacional, analítico, de tipo casos y controles. Se eligieron a los pacientes mayores de 18 años que asistieron a las IPS de Imsalud de una parte de la Zona Atalaya (Unidad Básica Comuneros, Niña Ceci, Ospina Pérez, Palmeras, Ermita, Olivos y Claret), desde enero de 2013 a diciembre de 2015. No eran elegibles aquellos pacientes menores de 18 años que tenían diagnóstico de TB extrapulmonar y VIH/sida, con lo cual se obtuvieron 28015 pacientes. Se identificaron 100 pacientes con diagnóstico de TB pulmonar, quienes correspondían al grupo de casos, y 27915 pacientes control que no desarrollaron TB pulmonar.

A los 100 pacientes con diagnóstico de TB pulmonar se les revisó sus respectivas historias clínicasy se extrajo la información correspondiente al número de identificación, sexo, edad y fecha de diagnóstico de la enfermedad, a partir de las fichas de notificación obligatoria. Se verificó, además, cuántos de estos pacientes tenían diagnóstico de DM2 como comorbilidad asociada, tras lo cual se encontraron 17 pacientes que presentaban ambas enfermedades.
El consolidado de pacientes diabéticos (847 pacientes) y los controles (27 915 pacientes) se obtuvo a partir de la base de datos de Imsalud. Los datos obtenidos se tabularon y analizaron en el programa SPSS.

\section{Resultados}

Se estudió una población de 28015 pacientes que asistían al servicio de consulta externa en las IPS pertenecientes a Imsalud de la Zona Atalaya (Clareth, Ospina Pérez, Palmeras, Comuneros, Ermita, Niña Ceci y Olivos), de los cuales 990 (32,1\%) corresponden a la población masculina y $19025(67,9 \%)$ a la población femenina (figura 1). La distribución por edad se especifica en la tabla 1.

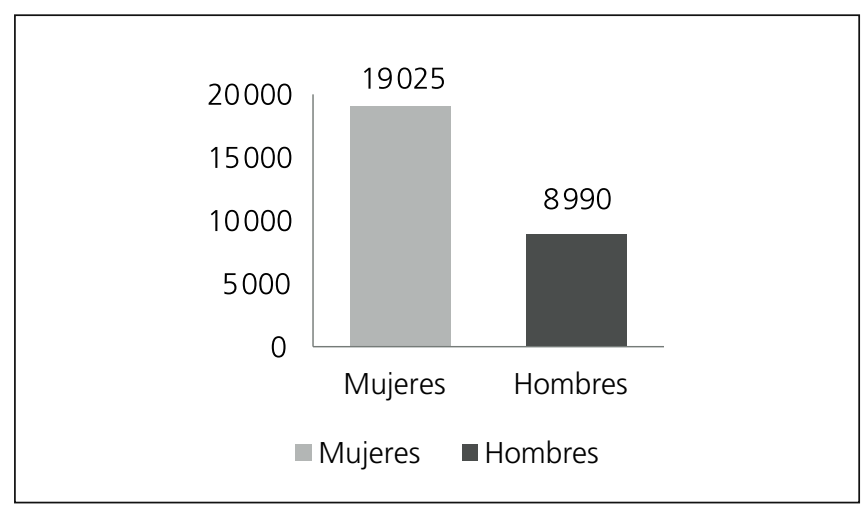

Figura 1.wFuente: Elaboración propia.

Tabla 1. Descripción de la población total según edad

\begin{tabular}{|c|c|c|}
\hline $\begin{array}{c}\text { Rango de edad } \\
\text { (años) }\end{array}$ & $\mathbf{n}$ & Porcentaje (\%) \\
\hline $18-20$ & 2526 & 9,02 \\
\hline $21-30$ & 5569 & 19,88 \\
\hline $31-40$ & 4658 & 16,63 \\
\hline $41-50$ & 4622 & 16,50 \\
\hline $51-60$ & 4140 & 14,8 \\
\hline$>61$ & 6500 & 23,2 \\
\hline Total & $\mathbf{2 8 ~ 0 1 5}$ & $\mathbf{1 0 0}$ \\
\hline
\end{tabular}

Fuente: Elaboración propia.

A partir de la población total se obtuvo una muestra de $100(0,4 \%)$ pacientes con diagnóstico de TB pulmonar, 
de los cuales $71(71 \%)$ corresponden a la población masculina y 29 (29\%) a la población femenina (figura 2). La distribución por edad se especifica en la tabla 2.

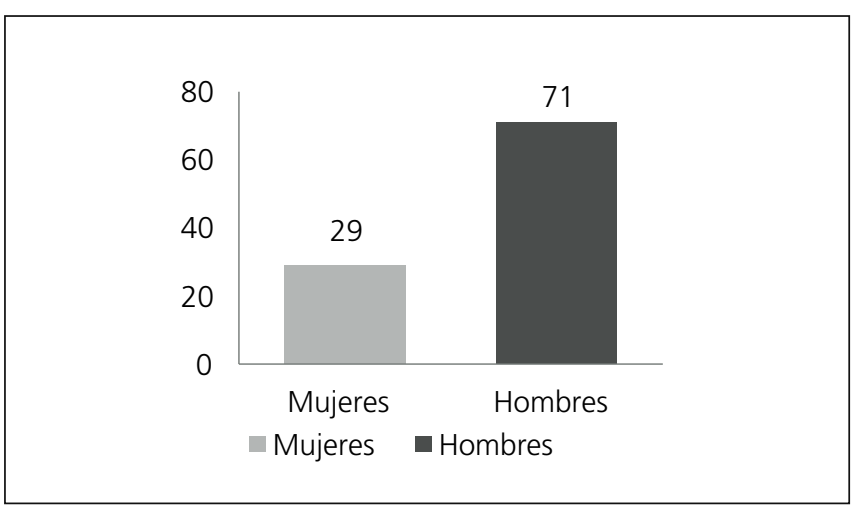

Figura 2. Caracterización de la población con TB pulmonar según sexo.

Fuente: Elaboración propia.

Tabla 2. Descripción de la población con TB pulmonar según edad

\begin{tabular}{|c|c|c|}
\hline $\begin{array}{c}\text { Rango de edad } \\
\text { (años) }\end{array}$ & $\mathbf{n}$ & Porcentaje (\%) \\
\hline $18-28$ & 3 & 3 \\
\hline $29-39$ & 19 & 19 \\
\hline $40-50$ & 14 & 14 \\
\hline $51-61$ & 16 & 16 \\
\hline $62-72$ & 27 & 27 \\
\hline $73-83$ & 15 & 16 \\
\hline$>84$ & 6 & 6 \\
\hline Total & $\mathbf{1 0 0}$ & $\mathbf{1 0 0}$ \\
\hline
\end{tabular}

Fuente: Elaboración propia.

La edad media de los pacientes con TB pulmonar fue de 47,68 años. Entre los grupos etarios, los de mayor frecuencia (27\%) correspondieron a los pacientes de 62 a 72 años (tabla 2); el paciente con mayor edad tenía 84 años y el de menor edad 18 años.

Simultáneamente, de la población total se obtuvo una muestra de 847 (3\%) pacientes con diagnóstico de DM2, de los cuales $252(29,75 \%)$ corresponden a la población masculina y 595 (70,25 \%) a la población femenina (figura 3). La distribución por edad se especifica en la tabla 3.

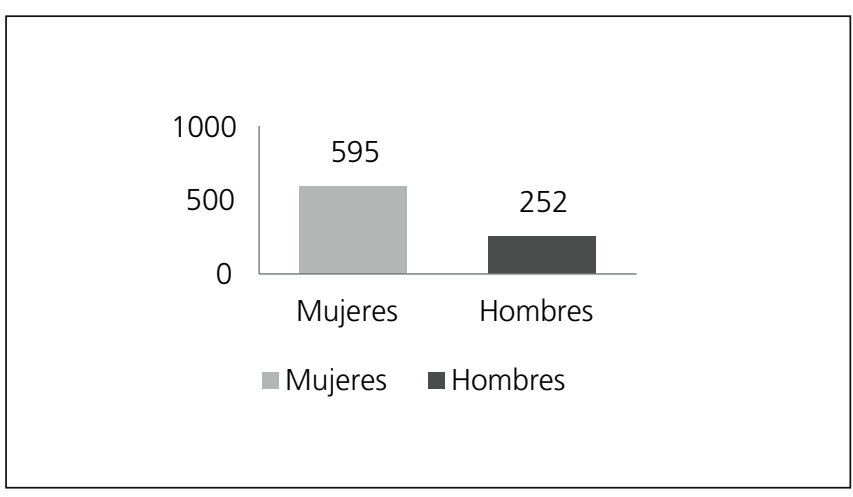

Figura 3. Caracterización de la población con DM2 según sexo.

Fuente: Elaboración propia.

Tabla 3. Descripción de la población con DM2 según edad

\begin{tabular}{|c|c|c|}
\hline $\begin{array}{c}\text { Rango de edad } \\
\text { (años) }\end{array}$ & N & Porcentaje (\%) \\
\hline $21-31$ & 4 & 0,4 \\
\hline $32-42$ & 38 & 4,5 \\
\hline $43-53$ & 166 & 19,6 \\
\hline $54-64$ & 273 & 32,3 \\
\hline$>65$ & 366 & 43,2 \\
\hline Total & $\mathbf{8 4 7}$ & $\mathbf{1 0 0}$ \\
\hline
\end{tabular}

Fuente: Elaboración propia.

Realizando la caracterización de los pacientes con DM2, se encontró que la edad media fue de 62 años. Entre los grupos etarios, los de mayor frecuencia $(43,2 \%)$ correspondieron a los de mayores de 64 años (tabla 3); el paciente con mayor edad tenía 94 años y el de menor edad 21 años.

Con respecto a la asociación DM2-TB, se encontraron $17(0,1 \%)$ pacientes que presentan el binomio, que fueron caracterizados por sexo, lo que arrojó un resultado de $7(41,17 \%)$ y $10(58,82 \%)$ pacientes de población femenina y masculina, respectivamente (figura 4). En la tabla 4, podemos encontrar la distribución de la asociación por grupos etarios. 


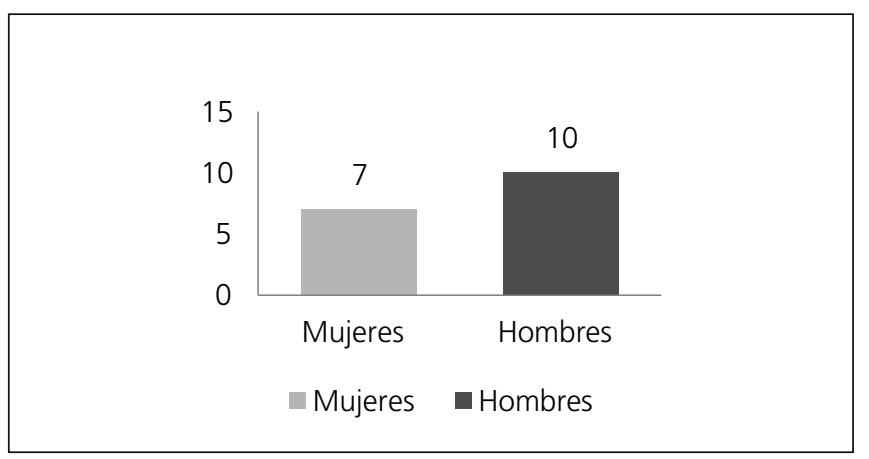

Figura 4. Caracterización de la población con TB pulmonar y DM2 según sexo.

Fuente: Elaboración propia.

Tabla 4. Descripción de la población con TB pulmonar y DM2 según edad

\begin{tabular}{|c|c|c|}
\hline $\begin{array}{c}\text { Rango de edad } \\
\text { (años) }\end{array}$ & N & Porcentaje (\%) \\
\hline $30-40$ & 1 & 5,9 \\
\hline $41-50$ & 5 & 29,41 \\
\hline $51-60$ & 7 & 41,17 \\
\hline$>61$ & 4 & 23,53 \\
\hline Total & $\mathbf{1 7}$ & $\mathbf{1 0 0}$ \\
\hline
\end{tabular}

Fuente: Elaboración propia.

En la asociación, la edad media fue de 54,05 años. Entre los grupos etarios, los de mayor frecuencia $(41,17 \%)$ correspondieron a los de 51 a 60 años (tabla 4); el paciente con mayor edad tenía 76 años y el de menor edad 38 años.

Con respecto a las medidas de asociación, se obtuvo una razón de ocurrencia (OR, por sus siglas en inglés) de 6,7 $y$ un chi cuadrado de 66,8 para una $\mathrm{p}<0,05$ con un intervalo de confianza del $95 \%(5,1-8,3)$ (tabla 5).

Tabla 5. Tabla de contingencia DM2-TB pulmonar

\begin{tabular}{|c|c|c|c|c|}
\hline \multirow{2}{*}{} & \multicolumn{4}{|c|}{ TB pulmonar } \\
\cline { 2 - 5 } & & Sí & No & Total \\
\cline { 2 - 5 } & Sí & 17 & 830 & 847 \\
\cline { 2 - 5 } & No & 83 & 27085 & 27168 \\
\cline { 2 - 5 } & Total & 100 & 27915 & 28015 \\
\cline { 2 - 5 } & \multicolumn{5}{|c}{ OR } & $\frac{17 \times 27085}{830 \times 83}=6,7$ \\
\hline
\end{tabular}

Fuente: Elaboración propia.

\section{Discusión}

La diabetes es una enfermedad crónica y degenerativa cuyo pronóstico evolutivo no solo depende de un diagnóstico oportuno, sino de un buen control glicémico por parte del paciente y del médico tratante, además, se deben implementar estilos de vida saludables.

Sin embargo, el estado de inmunosupresión al que conlleva esta enfermedad favorece el desarrollo de múltiples complicaciones y predispone la aparición de infecciones, tales como la TB.

La incidencia de TB aumenta con la edad, así como la DM tiene mayor prevalencia a mayor edad. De acuerdo con lo anterior, la edad puede actuar como variable de confusión (11). En este estudio, el rango de edades de mayor de presentación de DM y TB pulmonar fue de 62 a 72 años y $>63$ años, respectivamente.

Con los resultados obtenidos, se concluye que la DM2 se establece como factor de riesgo para adquirir TB. La razón de ocurrencia $(\mathrm{OR})$ versus no ocurrencia de $\mathrm{TB}$ pulmonar fue 6,7 veces mayor en pacientes con DM2, respecto de pacientes sin esta enfermedad. Esta asociación es estadísticamente significativa, lo que confirma hallazgos similares en la literatura.

Jeon et al. (1), mediante una revisión sistemática y un metaanálisis de estudios observacionales, evaluaron la asociación DM/TB ajustado por la edad, tras lo cual encontraron que la DM se asoció con un mayor riesgo de TB. Asimismo, en Chile, Ramonda et al. (9) evidenciaron una fuerte asociación entre ambas enfermedades (OR 2,3 ajustado por edad y nivel socioeconómico), mediante el diseño de casos y controles, de 2006 a 2009 en el Servicio de Salud Metropolitano Sur. De igual forma, el estudio demostró que los diabéticos se constituían en un grupo de riesgo para adquirir TB, especialmente las mujeres (9), a diferencia del presente estudio, que evidenció mayor riesgo en la población masculina.

Unos de los pocos estudios realizados en el continente africano, en Túnez (1), en personas hospitalizadas por $\mathrm{TB}$, mostraron que la asociación DM/TB estuvo presente en el $7,6 \%$.

En Suecia, Oscarsson y Silwer (1) constataron la TB pulmonar en el 3,6\% de los enfermos con DM, comparado con un $0,88 \%$ en un grupo control de la población en general. En este estudio, se evidenció que la TB pulmonar se presentó en el $2 \%$ de los pacientes diabéticos frente a un $0,3 \%$ en el grupo control. 
En la literatura revisada, se planteó la mayor susceptibilidad presentada en personas con DM de contraer TB, por defectos en la inmunidad celular tanto innata como adaptativa, causados por periodos de hiperglicemia, además de la alteración en las funciones de los leucocitos polimorfonucleares (12).

Finalmente, la falta de información sistematizada en una base de datos histórica sobre la enfermedad, que es una de las grandes limitaciones para el desarrollo de este tipo de estudios. Es escasa la información registrada en las historias clínicas, que carecen de los soportes paraclínicos.

\section{Conclusión}

El estudio mostró características relevantes en la población, que asocian las dos enfermedades. En la zona de influencia analizada, la población masculina $(58,82 \%)$ constituyó la población predominante de diabéticos (70,25\%), lo que puede sugerir que tiene mayor probabilidad de desarrollar TB.

El rango de edad en el que se presentó el mayor porcentaje de esta asociación fue entre 51 y 60 años $(41,17 \%)$, lo que confirma una relación directamente proporcional entre la edad y el mayor riesgo de padecer estas enfermedades.

La prevalencia de $\mathrm{DM}$ en los pacientes puede reducir la eficacia de las acciones preventivas en la TB, por una mayor probabilidad de adquirir infecciones. Con un chi cuadrado de 66,8 para una $p<0,05$, el riesgo de presentar TB en los pacientes diabéticos fue 6,7 veces mayor.

Por tanto, la DM2 se considera un factor de riesgo importante para el desarrollo de TB. Por lo anterior, se recomendaría realizar un nuevo estudio que involucre una población mayor que permita generar impacto en la salud pública integral mediante el desarrollo de programas preventivos particulares en el país, así como la revisión de buenas prácticas en otras regiones del mundo. Esto implica no solo la atención de la población vulnerable a esta enfermedad, sino la inclusión de campañas que promuevan estilos de vida saludables desde los primeros años de vida.

\section{Agradecimientos}

Este artículo fue desarrollado gracias a la Universidad de Pamplona, a través del Área de Electiva e Investigación, bajo la dirección de la doctora Maritza Maldonado y la tutoría de los doctores Miguel Alfonso Chahin y Germán Wilches, quienes desde el primer momento nos guiaron y asesoraron para llevar a feliz término nuestro problema de investigación. Finalmente, agradecimiento especial a la ESE Imsalud que nos proporcionó toda la información necesaria de los pacientes en estudio.

\section{Referencias}

1. Arnold Y, Castelo L, Licea M. Diabetes mellitus y tuberculosis. Rev Peru Epidemiol. [internet] 2012 [citado $2019 \mathrm{mrz}$ 16]; 16(2):1-8. Disponible en: https://www.redalyc.org/ html/2031/203124632003/

2. Longo DL. Harrison: principios de medicina interna. $18 .^{a}$ ed. México: McGraw Hill; 2012.

3. Martínez-Pulgarín DF, Rico-Gallego JA, Vera-Polanía F, et al. Comorbilidad de tuberculosis y diabetes en un hospital de Colombia. Rev Peru Med Exp Salud Pública. [internet] 2014 [citado $2019 \mathrm{mrz}$ 16];31(1):397-98. Disponible en: https://www.scielosp.org/article/ssm/content/raw/?resource_ssm_path=/media/assets/rpmesp/ v31n2/a37v31n2.pdf

4. Instituto Nacional de Salud. Informe del evento de tuberculosis a periodo epidemiológico XII de 2014. Bogotá: Instituto Nacional de Salud; 2014.

5. Instituto Nacional de Salud. Informe del evento de tuberculosis hasta el periodo epidemiológico VII de 2016. Bogotá: Instituto Nacional de Salud; 2016.

6. Murray S, Shaw J, Sicree R, et al. La epidemia de diabetes en crecimiento: predecir el futuro. Diabetes Voice. [internet] 2003 [citado $2019 \mathrm{mrz}$ 16];48(1):12-16. Disponible en: https://docplayer.es/10715257-La-epidemia-de-diabetes-en-crecimiento-predecir-el-futuro.html

7. Observatorio Nacional de Salud. Carga de enfermedad por enfermedades crónicas no transmisibles y discapacidad en Colombia: V edición. Bogotá: Observatorio Nacional de Salud; 2015.

8. Salazar MA, Sotelo N. Tuberculosis y diabetes mellitus en la jurisdicción sanitaria número 2 del estado de Guerrero: una comunicación breve de un estudio descriptivo. Neumol Cir Torax. 2011;70(3):152-156. Disponible en: http://www.medigraphic.com/cgi-bin/new/resumen. cgi? IDARTICULO=31095

9. Herrera T, Leiva E, Martín F, et al. Asociación entre tuberculosis y diabetes mellitus en la región metropolitana. Rev Chil Enf Respir. 2013;29(3):171-175. http://dx.doi. org/10.4067/S0717-73482013000300008 
10. Mendoza León C. Diabetes mellitus mal controlada como factor de riesgo para tuberculosis resistente en el Hospital Nacional Daniel Alcides Carrión durante 2010-2012. Interciencia. [internet] 2014 [citado $2019 \mathrm{mrz}$ 16];5(1):3540. Disponible en: http://ateneo.unmsm.edu.pe/ateneo/ handle/123456789/3080

11. Méndez J, Sánchez É, Saavedra A, et al. Prevalencia de tuberculosis latente en pacientes con diabetes mellitus en una institución hospitalaria en la ciudad de Bogotá, Colombia: un estudio de corte transversal. Acta Med Colomb. [internet] 2017 [citado $2019 \mathrm{mrz}$ 16];42(3):165-171. Disponible en: http://www.scielo.org.co/scielo.php?pid=S0120$24482017000300165 \&$ script $=$ sci_abstract\&tlng=en
12. Ugarte-Gil C, Moore DA. Comorbilidad de tuberculosis y diabetes: problema aún sin resolver. Rev Peru Med Exp Salud Publica. [internet] 2014 [citado $2019 \mathrm{mrz}$ 16]; 31(1):137-142. Disponible en: https://www.scielosp.org/ article/ssm/content/raw/?resource_ssm_path=/media/ assets/rpmesp/v31n1/a20v31n1.pdf

13. Organización Mundial de la Salud. Informe mundial sobre la tuberculosis 2014. [internet] 2014. Disponible en: https://www.who.int/tb/publications/global_report/ gtbr14_execsummary_summary_es.pdf 